\title{
Reduction methods and chaos for quadratic systems of differential equations
}

\author{
Sergey Vakulenko, Dmitry Grigoriev, and Andreas Weber
}

\begin{abstract}
We consider systems of differential equations with quadratic nonlinearities having applications for biochemistry and population dynamics, which may have a large dimension $n$. Due to the complexity of these systems, reduction algorithms play a crucial role in study of their large time behaviour. Our approach aims to reduce a large system to a smaller one consisting of $m$ differential equations, where $m<<n$. Under some restrictions (that allow us to separate slow and fast variables in the system) we obtain a new system of differential equations, involving slow variables only. This reduction is feasible from a computational point of view for large $n$ that allows us to investigate sensitivity of dynamics with respect to random variations of parameters. We show that the quadratic systems are capable to generate all kinds of structurally stable dynamics including chaos.
\end{abstract}

\section{Introduction}

In this paper, we consider the dynamics a class of systems of ordinary autonomous differential equations with quadratic nonlinearities. These systems may have applications for population dynamics and biochemistry (many examples from the BioModel database [20] belong to the considered class). We develop algebraic methods to investigate the dynamics of these systems, which use elementary algebra and invariant manifold theory and do not involve numerical integration of differential equations. They are capable to find equilibria, invariant domains and absorbing sets. We reduce high-dimensional dynamics to low dimensional ones. We prove that this class of the quadratic systems can generate all kinds of structurally stable dynamics, including chaotic ones.

1.1. A class of quadratic systems. In chemical and biological applications, the species concentrations satisfy ordinary differential equations:

$$
\frac{d x_{i}}{d t}=F_{i}(\boldsymbol{x}), \quad \boldsymbol{x} \in \mathbf{R}^{n}, 1 \leq i \leq n .
$$

where $F_{i}$ are some smooth functions of the concentrations $x_{i}$.

We focus our attention on a special class QS of systems (1.1), where the field $F$ in (1.1) is defined by

$$
F_{i}=b_{i}+\sum_{j \in[1, \ldots, n], j \neq i} M_{i j} x_{j}-\left(\tilde{b}_{i}+\sum_{k=1}^{n} b_{i k} x_{k}\right) x_{i}+\sum_{j, k \in[1, \ldots, n], j \neq i, k \neq i} \sum K_{i j k} x_{j} x_{k},
$$

2010 Mathematics Subject Classification. 14T05, 92C42. 
where $i=1, \ldots, n$ and

$$
b_{i} \geq 0, \tilde{b}_{i} \geq 0, M_{i j} \geq 0, K_{i j k} \geq 0 .
$$

Such systems are often arise in biology and biochemistry (for biochemical applications see BioModel database [20]). Note that in the case when all coefficients $b_{i}, M_{i j}$ and $K_{i j k}$ equal zero, system (1.1) reduces to a generalized Lotka-Volterra system [10]. In the BioModel database many systems are defined by the vector fields having the form (1.2). Some of these systems are linear and many others do not contain terms $K_{i j k} x_{j} x_{k}$ with $j \neq i, k \neq i$.

1.2. Large time behaviour and structural stability. In this paper, we focus on the multidimensional systems, where the number of the species (reagents) is large, $n>>1$. We consider the following question: what large time behaviour can emerge in a system from the class QS when its coefficients range a large domain. In fact, the kinetic rates (in our case, coefficients $b_{i k}, M_{i j}, \tilde{b}_{i}$ and $K_{i j k}$ ) are generally highly uncertain. This uncertainty arises as a result of difficulties in measuring of these parameters and a complicated structure of biochemical and biological systems for large $n$. Therefore, sensitivity with respect to kinetic rate variations is a crucial question. A review of the numerical methods that allow us to estimate sensitivity, can be found in [37].

The sensitivity problem is connected with the other question, namely, the structural stabilityof dynamical systems. The fundamental concept of structural stability was introduced by A. Andronov and L. Pontryagin in 1937 [1]. Roughly speaking, this means that small perturbations of a structurally stable system do not change the topological structure of the system trajectories.

Definition 1.1. We say that a dynamical system $S^{t}$ on $X$ is topologically orbitally equivalent to a dynamical system $T^{t}$ on $Y$ if there is a homeomorphism $h: X \rightarrow Y$ which preserves orbits and the sense of direction in time.

Remark: One can use less restrictive definitions when $h$ is a homeomorphism connecting the corresponding attractors or the corresponding non-wandering sets, or neighborhoods of the attractors.

Definition 1.2. We say that a global semiflow $S^{t}$ on a compact smooth manifold $\mathcal{M}$ defined by differential equations

$$
\frac{d q}{d t}=Q(q), \quad q \in \mathcal{M}
$$

is structurally stable if each perturbed field $Q+\tilde{Q}$ such that

$$
|\tilde{Q}|_{C_{\mathcal{M}}^{1}}<\epsilon
$$

generates a semiflow that is orbitally topologically equivalent to $S^{t}$, if $\epsilon>0$ is small enough.

For two dimensional case $n=2$ we have fundamental theorems of M. Peixoto [23], which show that here the situation is relatively simple. Namely, a vector field on a two dimensional smooth compact manifold (a closed surface, for example) is structurally stable if and only if this field is of the Morse-Smale type. Remind that dynamics of Morse-Smale systems is relatively simple and does not exhibit chaotic phenomena. Moreover, for any integer $r \geq 1$ the set of the Morse-Smale fields of class $C^{r}$ is open and dense in the set of all $C^{r}$ vector fields, i.e., a generic system in $2 D$ belongs to the Morse-Smale class (see , for example, $[\mathbf{1 6}]$ for more detail). 
However, for $n>2$ the theory of dynamical systems faces formidable problems. It is impossible to find a classification, up to homeomorphisms, of finite dimensional dynamical systems. This fact follows from the fundamental results due to S. Smale: if the dimension $n>3$, then there is a structurally stable system that is not Morse-Smale and the set of structurally stable fields of class $C^{r}$ is not open and dense in the set of all $C^{r}$ vector fields (see [16], Chapter 1, Theorem 1.6). Therefore, it is impossible to construct a "general theory" of dynamical systems.

To circumvent these serious difficulties, connected with the structural instability and the uncertainty in kinetic rates, we propose a reduction approach combining an algebra and a special method, the realization of vector fields (RVF) $[\mathbf{2 4}, \mathbf{2 5}]$. Due to the complexity of biochemical reaction networks, reduction algorithms play a crucial role in study of biochemical system large time behaviour. Our approach aims to reduce a large system to a smaller one consisting of $m$ differential equations, where $m<<n$. Namely, under some restrictions (that allow us to separate $m$ slow and $n-m$ fast variables in the system) we obtain a new system of differential equations involving the slow variables only. The vector field, that defines the right hand sides of the reduced slow system, is called the inertial form [35]. This reduction is feasible from a computational point of view for large $n$. An interesting fact is as follows. We show that this inertial form is robust for large systems with respect to random variations of many system parameters but it may be sensitive with respect to other variations. Therefore we are capable to pick out some key parameters.

Note that there also exist other methods for the reduction of quadratic systems. In the paper [13] algebraic invariants were found, which allow to test whether a given polynomial kinetic system can be reduced, by a change of variables to the Lorenz system, or not. General (not obligatory kinetic) systems of differential equations with quadratic nonlinearities were investigated in detail by algebraic methods in the work [18]. It was shown that they can generate all trajectories that can be obtained by arbitrary polynomial differential equations of any orders

$$
\left(\frac{d}{d t}\right)^{n} y=P\left(y, \frac{d y}{d t}, \ldots,\left(\frac{d}{d t}\right)^{n-1} y\right)
$$

where $P$ is a polynomial.

We also show that a narrow class of systems with quadratic nonlinearities can generate all possible structurally stable dynamics. To this end we use algebraic methods, the invariant manifold theory (decomposition into slow and fast variables) $[\mathbf{7}, \mathbf{1 4}, \mathbf{1 6}, \mathbf{2 8}$, $35,40,43]$ and the RVF method $[24,25,26,27,31,39]$. In order to obtain a given dynamics, we vary some parameters in equations. In our case the parameters are entries of the matrix $\mathbf{M}$ that defines linear terms in the right hand sides of the equations. The main difference between results $[\mathbf{1 8}, \mathbf{1 3}]$ and this paper is as follows. In order to obtain a given dynamics by methods $[\mathbf{1 8}, \mathbf{1 3}]$ it is necessary to vary all coefficients whereas we adjust only a part of coefficients corresponding to linear terms (the quadratic terms are fixed).

\section{Some definitions and preliminaries}

2.1. Positive cones and cooperative systems. It is natural to assume, from chemical and biological points of view, that $\boldsymbol{x}(t)$ should stay in the positive cone $\mathbf{R}_{>}^{n}=\{\boldsymbol{x}$ : $\left.x_{i} \geq 0, i=1, \ldots, n\right\}$. We assume that a local semiflow $S^{t}$, generated by system (1.1), 
conserves the positive cone, i.e., $\boldsymbol{x}(0) \in \mathbf{R}_{>}^{n}$ implies $\boldsymbol{x}(t) \in \mathbf{R}_{>}^{n}$ for all $t$ while the trajectory $\boldsymbol{x}(t)$ is defined. Then the positive cone can be considered as a phase space $H$ of our semiflow. For systems of chemical kinetics, fairly general conditions that guarantee this cone preservation property, were obtained by A. Volpert [42]. In the case of QS systems we have an elementary lemma that follows from results $[\mathbf{4 2}]$ and $[\mathbf{3 4}]$.

LEMmA 2.1. If conditions (1.3) holds, then the semiflow defined by system (1.1) with the right hand sides (1.2) preserves the positive cone $\mathbf{R}_{>}^{n}$.

Given an $F_{i}$, it is difficult to describe in detail the local semidynamical system $S^{t}$ associated with this vector field. Some facts are known about so-called monotone dynamical systems. To describe such systems let us introduce a partial order in $\mathbf{R}^{n}$ :

$$
u \geq v
$$

means that for all

$$
u_{i} \geq v_{i}
$$

for all $i=1, \ldots, n$. Similarly, we introduce $u>v$. Some local semiflows preserve this partial order $\geq$ and they are called monotone dynamical systems. The monotone semiflows have an important property: all local attractors are equilibria and a complicated large time behaviour is possible only for some specially adjusted initial data (data from a set of measure 0 , see $[\mathbf{1 5}, \mathbf{3 3}]$ and $[\mathbf{2 7}]$ for an overview). An important class of the monotone dynamics appears if we consider so-called cooperative systems, where

$$
\frac{\partial F_{i}}{\partial x_{j}} \geq 0 \quad \forall i \neq j, \quad\left(x_{1}, \ldots, x_{n}\right) \in \mathbf{R}_{>}^{n} .
$$

Then $u(0) \geq v(0)$ implies $u(t) \geq v(t)$. Note that the QS systems defined by (1.2) are cooperative if (1.3) holds and $b_{i k} \leq 0$.

Unfortunately, a number of biochemical systems are not cooperative. Monotone systems that arise in chemical kinetics were studied in [19].

2.2. Invariant manifolds and attractors. To simplify the statement, we formulate here some main definitions from dynamical system theory. If solutions of (1.1) exist for all times $t$, our evolution equation defines a global semiflow (semigroup). It is a family of maps $S^{t}$ such that

$$
\begin{gathered}
(i) \quad S^{0}=I \\
(i i) \quad S^{t+\tau}=S^{t} S^{\tau}, \forall t, \tau \in \mathbf{R}^{+}, \\
\text {(iii) } \quad S^{t} \in C^{0}\left(\mathbf{R}^{n}, \mathbf{R}^{n}\right) \quad \forall t>0 .
\end{gathered}
$$

However, even a simple system with quadratic terms does not define, in general, a global semiflow. As an example, one can consider the simplest equation $u^{\prime}=u^{2}$, which exhibits a blow-up effect where solutions are defined only within bounded time intervals (then we speak about a local semiflow). Other examples and a general approach for blow-up effect can be found in [6]. So, checking global semiflow property may be a non trivial problem. We can do it using so-called invariant domains (see Sect. 2.2). In the general case eqs. (1.1) define a local semiflow.

If we have established that the system generates a global semiflow $S^{t}$, the next step is to check that the system is dissipative. A global semiflow $S^{t}$ is dissipative, if there is an 
absorbing set $D \subset \mathbf{R}_{>}^{n}$, i.e., a set such that each trajectory $\boldsymbol{x}(t), t \in[0,+\infty)$ enters this set at some moment $T_{0}(\boldsymbol{x}(0))$ and does not leave this set in all following time moments:

$$
\boldsymbol{x}(t)=S^{t} \boldsymbol{x}(0) \in D \quad \forall t>T_{0} .
$$

Some criterion for dissipativity we state in this paper. If system (1.1) is dissipative, we can assert that this system (global semiflow) possesses a global attractor, which is a compact, connected and invariant set $[\mathbf{1 2}]$. To define a global attractor, we first define globally attracting sets. We say that a set $A$ is a globally attracting one, if for each open neighborhood $V(A)$ of $A$ and each bounded set $B$ there is a time moment $T(V, B)$ such that

$$
\boldsymbol{x}(t)=S^{t} \boldsymbol{x}(0) \in V \quad \forall t>T(V, B) \quad \forall x(0) \in B .
$$

The invariant set $I$ can be defined as a set such that if a trajectory $\boldsymbol{x}(t), t \in(-\infty,+\infty)$ lies in $I$ at $t=0$, this trajectory stays in $I$ for all time moments:

$$
\boldsymbol{x}(0) \in I \Longrightarrow \boldsymbol{x}(t) \in I, \quad t \in(-\infty,+\infty) .
$$

A set is positively invariant, if the last inclusion holds only for $t>0$, and locally invariant if it holds for some $t \in(-\delta,+\delta)$. The global attractor can be defined as a minimal closed globally attracting invariant set. Note that there is a variation in attractor definition. Sometimes one uses other definitions, for example, J.Milnor's attractor $[\mathbf{1 6 , 1 7 , ~ 2 8 , ~ 4 1 ]}$.

We say that a set $A_{1}$ is a locally attracting one, if there is $B_{1}(A)$ (attraction basin) such that for each open neighborhood $V\left(A_{1}\right) \subset B_{1}$ of $A_{1}$ and each bounded set $B \subset B_{1}$ there is a time moment $T(V, B)$ such that

$$
\boldsymbol{x}(t)=S^{t} \boldsymbol{x}(0) \in V \quad \forall t>T(V, B) \forall x(0) \in B .
$$

The local attractor is a minimal closed locally attracting invariant set in $B_{1}$.

Invariant manifolds, locally attracting invariant manifolds, positively invariant manifolds are, respectively, sets $I$, which enjoy the corresponding property and, moreover, are smooth manifolds. Importance of locally attracting invariant smooth manifolds can be explained as follows. If we could find a locally (or even globally) attracting invariant manifold $M \subset D$ of a low dimension $m<<n$, all dynamics in $D$ can be reduced to a dynamics on $M$ defined by a smooth vector field (an inertial form) of dimension $m$. However, an analytic expression for this field may be more complicated, for example, if the original dynamics is defined by a polynomial field, the inertial form can be defined by a rational in $x_{i}$ functions (the well known example: the Michaelis-Menten dynamics can be obtained from a polynomial dynamics).

2.3. Hyperbolic sets and chaos. In this subsection we consider some facts on hyperbolic sets used below. We follow [29] and first consider dynamical systems with discrete time, i.e., maps. They can appear as numerical realizations of (1.1). Roughly speaking, a subset $\Gamma$ of a smooth manifold $\mathrm{M}$ is said to have a hyperbolic structure with respect to a smooth map $f$ if its tangent bundle may be split into two invariant subbundles, one of which is contracting and the other is expanding under $f$ (see in monograph [29] for more detail). In the case of flows we can use a similar definition [29].

For flows, the simplest examples of hyperbolic sets are hyperbolic equilibria and hyperbolic limit cycles. There are possible hyperbolic sets having a fractal structure or smooth (normally hyperbolic manifolds) but with a complicated dynamics (see for example, $[\mathbf{2}, \mathbf{1 1}, \mathbf{1 7}, \mathbf{2 9}, \mathbf{2 8}]$. They also are structurally stable (persistent). 
To prove, in a mathematically rigorous way, that a concrete system of polynomial equations generates a chaotic behavior (i.e., the attractor is not a finite union of smooth manifolds), it is not an easy problem. If we use numerical simulations, the question arises on numerical errors and how to distinguish random fluctuations from chaos. Even such classical system, as the Lorenz system [21], studied in a number of works, is not understood completely (where $n=3$ !). A computer assisted proof of chaos existence for the Lorenz system see [36] and for the forced damped pendulum equation [5].

Reference remark: The question of numerical checking the Morse-Smale property was studied by B. M. Garay [8]. The general review for numerical approximation of attractors etc., see in monograph L. Grune [9]. Note that numerical approximations are correct if our system is structurally stable. Some topological methods for the Morse-Smale systems can be found in $[\mathbf{3 2}]$.

\section{Almost cooperative systems}

Let us define a new class of systems of differential equations, almost cooperative systems (AC systems). This definition is motivated by the fact that if in relations (1.2) coefficients $b_{i k}>0$ for some $i$ and $k$, then the corresponding system (1.1) is not cooperative, however, the system can lie in the AC class. For general AC systems the attractor structure is unknown, but we can obtain some estimates for their solutions.

DeFINITION 3.1. Let us consider a smooth vector field $F(\boldsymbol{x})$ defined on an open subset $D$ of the positive cone $\mathbf{R}_{>}^{n}$. We say that it is a lower almost cooperative (AC) field in $D$ if

$$
F_{i}(\boldsymbol{x})=G_{i}(\boldsymbol{x})-x_{i} q_{i}(\boldsymbol{x}),
$$

where $G$ is bounded by smooth cooperative field $G^{+}$, i.e.,

$$
G_{i}(\boldsymbol{x}) \leq G_{i}^{+}(\boldsymbol{y}), \quad \forall x, y \in D, x \leq y,
$$

where

$$
\frac{\partial G_{i}^{+}}{\partial x_{j}} \geq 0 \quad \forall i \neq j
$$

and $q_{i}$ satisfy estimates

$$
q_{i}(\boldsymbol{x})>q_{i}^{-}(\boldsymbol{y}), \quad \forall x, y \in D, x \leq y
$$

for some functions $q_{i}^{-}$. We say that $F$ is an upper almost cooperative field if relation (3.1) holds and there are a smooth field $G^{-}$and smooth functions $q_{j}^{+}$such that

$$
\frac{\partial G_{i}^{-}}{\partial x_{j}} \geq 0 \quad \forall i \neq j
$$

and

$$
\begin{gathered}
G_{i}(\boldsymbol{x}) \geq G_{i}^{-}(\boldsymbol{y}), \quad \forall x, y \in D, x \geq y, \\
q_{i}(\boldsymbol{x})<q_{i}^{+}(\boldsymbol{y}), \quad \forall x, y \in D, x \geq y .
\end{gathered}
$$

We say that $F$ is almost cooperative $(A C)$ if this field is upper and lower almost cooperative.

Example. Many systems from the BioModel database [20] lie in the AC class. For instance, let us consider the following system (see BioModel database, example 21)

$$
\frac{d x_{1}}{d t}=-\left(k_{0}+k_{8}\right) x_{1}+k_{2} x_{3}+k_{22}
$$




$$
\begin{gathered}
\frac{d x_{2}}{d t}=-\left(k_{1}+k_{9}\right) x_{2}+k_{3} x_{4}+k_{23}, \\
\frac{d x_{3}}{d t}=k_{0} x_{1}-\left(k_{2}+k_{4}+k_{10}\right) x_{3}+k_{6} x_{5}, \\
\frac{d x_{4}}{d t}=k_{1} x_{2}-\left(k_{3}+k_{5}+k_{11}\right) x_{4}+k_{7} x_{6}, \\
\frac{d x_{5}}{d t}=k_{4} x_{3}-\left(k_{6}+k_{12}\right) x_{5}-k_{14} x_{5} x_{6}+k_{15} x_{7}, \\
\frac{d x_{6}}{d t}=k_{5} x_{4}-\left(k_{7}+k_{13}\right) x_{6}-k_{14} x_{5} x_{6}+k_{16} x_{7}, \\
\frac{d x_{7}}{d t}=k_{14} x_{5} x_{6}-\left(k_{15}+k_{16}+k_{18}\right) x_{7}+k_{17} x_{8}, \\
\frac{d x_{8}}{d t}=k_{16} x_{7}-\left(k_{17}+k_{19}\right) x_{8} .
\end{gathered}
$$

Notice that the semiflow defined by (3.6)-(3.13) preserves the positive cone. It is easy to see that this system is defined by a lower $\mathrm{AC}$ field if all $k_{i}>0$. Indeed, the components of the vector field $F$ that defines the right hand sides of equations (3.6)-(3.13) have the form $F_{i}=G_{i}(\boldsymbol{x})-x_{i} q_{i}(\boldsymbol{x})$, where the field $G$ is cooperative on the positive cone. Moreover, $q_{i}(\boldsymbol{x}) \geq 0$ for $\boldsymbol{x} \geq 0$. We can set $G_{i}^{ \pm}=G_{i}$ and $q_{i}^{ \pm}=$const, for example,

$$
q_{1}^{ \pm}=k_{0}+k_{8}, q_{2}^{ \pm}=k_{1}+k_{9}, q_{3}^{ \pm}=k_{2}+k_{4}+k_{10}, q_{4}^{ \pm}=k_{3}+k_{5}+k_{11},
$$

and

$$
q_{5}^{-}=k_{6}+k_{12}, q_{6}^{-}=k_{7}+k_{13}, q_{7}^{ \pm}=k_{15}+k_{16}+k_{18}, q_{8}^{ \pm}=k_{17}+k_{19}
$$

Below we shall show that under some conditions solutions $\boldsymbol{x}$ are bounded, i.e., $0<x_{i}<C_{i}$, where $C_{i}>0$ are some constants. Let us define $D=\left\{\boldsymbol{x}: 0<x_{i}<C_{i}\right\}$. Then we can set $q_{5}^{+}=k_{6}+k_{12}+k_{14} C_{5}$ and $q_{6}^{+}=k_{7}+k_{13}+k_{14} C_{6}$. Consequently, system (3.6)-(3.13) lies in $\mathrm{AC}$ class in the domain $D$.

Remark: For a general system of polynomial differential equations it is not quite obvious even that the system generates a global semiflow (i.e., solutions starting in an open domain $\Omega$ are defined for all $t>0$ ) and that, if this holds, then the system is dissipative. To verify a global semiflow existence, we can use the following criterion. Assume the boundary $\partial \Omega$ of $\Omega$ is a union of finite number of smooth manifolds, for example, a simplex $S$ or a box $\Pi$. We say that the domain $\Omega$ is invariant, if at the boundary $\partial \Omega$ the vector field $F$ is directed inward $\Omega[\mathbf{3 4}]$. It is well known that the system generates a global semiflow in $\Omega$, if $\Omega$ is invariant.

Let us formulate some useful sufficient conditions, which allow us to check that the dynamical system has an invariant domain. For systems of chemical kinetics such conditions were found by A. Volpert [42].

i If the system has a conservation law

$$
E(\boldsymbol{x})=\sum_{i=1}^{n} c_{i} x_{i}, \quad c_{i}>0
$$

and preserves the positive cone, the system defines a global semiflow in any simplex $S=$ $\left\{\boldsymbol{x}: x_{i} \geq 0, E(\boldsymbol{x})<C\right\}$. 
ii The same holds if $E(\boldsymbol{x})$ satisfies the inequality

$$
\frac{d E(\boldsymbol{x}(t)}{d t}<-a_{0} E(\boldsymbol{x}(t))+k_{0}, \quad a_{0}, k_{0}>0
$$

along all the trajectories $\boldsymbol{x}(t), t \geq 0$. In this case there exists an absorbing set $\mathcal{A}$ defined by $\mathcal{A}=\left\{\boldsymbol{x}: E(\boldsymbol{x})<k_{0} / a_{0}\right\}$ and the corresponding semiflow is dissipative,

iii if there exists an invariant box

$$
\Pi_{\boldsymbol{a}}=\left\{\boldsymbol{x}: 0 \leq x_{i} \leq a_{i}\right\}
$$

As an example, consider (3.6)-(3.13). Let $E$ be defined by (3.14), where $n=8$. Then $\frac{d E(\boldsymbol{x}(t))}{d t}=-b_{1} x_{1}-b_{2} x_{2}-b_{3} x_{3}-b_{4} x_{4}-b_{5} x_{5}-b_{6} x_{6}-b_{7} x_{7}-b_{8} x_{8}-k_{14} x_{5} x_{6}+k_{22}+k_{23}$, where

$b_{1}=c_{3} k_{0}-c_{1}\left(k_{0}+k_{8}\right), \quad b_{2}=c_{4} k_{1}-c_{2}\left(k_{1}+k_{9}\right), \quad b_{3}=c_{1} k_{2}+c_{5} k_{4}-c_{3}\left(k_{2}+k_{4}+k_{10}\right)$, $b_{4}=c_{6} k_{5}+c_{2} k_{3}-c_{4}\left(k_{3}+k_{5}+k_{11}\right), \quad b_{5}=c_{3} k_{6}-c_{5}\left(k_{6}+k_{12}\right), \quad b_{6}=c_{4} k_{7}-c_{6}\left(k_{7}+k_{13}\right)$ and

$$
b_{7}=c_{5} k_{15}+\left(c_{6}+c_{8}\right) k_{16}-c_{7}\left(k_{15}+k_{16}+k_{18}\right), \quad b_{8}=c_{7} k_{17}-c_{8}\left(k_{17}+k_{19}\right) .
$$

If all $b_{i}>0$ then relation (3.15) holds. Thus, in this case our system has globally bounded solutions and defines a global semiflow. This system has at least one equilibrium. We shall discuss the question on the number of equilibria for (3.6)-(3.13) in the next section. Note that conditions $b_{i}>0, c_{i}>0$ give a system of linear inequalities for $c_{i}$. In general, it is not clear that this system has a solution. However, if $k_{18}>k_{16}$, then we can set $c_{j}=1$ for all $j$.

For quadratic fields $F$, such as (3.6)-(3.13), checking the point iii that a $\Pi_{a}$ is invariant, can be simplified. Let us formulate an assertion.

Lemma 3.2. Let conditions (1.3) hold and $b_{i k} \geq 0$ for all $i, k$. If the vector $\boldsymbol{a}$ is a solution of the following quadratic programming problem:

$$
b_{i}+\sum_{j=1, j \neq i}^{n} M_{i j} a_{j}+\sum_{j, k \in[1, \ldots, n], j \neq i, k \neq i} \sum K_{i j k} a_{j} a_{k}<\left(\tilde{b}_{i}+b_{i i} a_{i}\right) a_{i}, \quad i=1, \ldots, n,
$$

then the box $\Pi_{\boldsymbol{a}}$ is invariant.

If $K_{i j k}=0$ then system of inequalities (3.16) reduces to a linear programming problem. Given a lower AC field $F$, we associate with this field the superfield $F^{+}$defined by

$$
F_{i}^{+}(\boldsymbol{x})=G_{i}(\boldsymbol{x})^{+}-x_{i} q_{i}^{-}(\boldsymbol{x}), \quad \forall x \in D .
$$

Similarly, given an upper AC field $F$, we associate the subfield $F^{+}$by

$$
F_{i}^{-}(\boldsymbol{x})=G_{i}(\boldsymbol{x})^{-}-x_{i} q_{i}^{+}(\boldsymbol{x}), \quad \forall x \in D .
$$

The following assertion allows us to estimate solutions of almost cooperative systems. 
Proposition 3.3. Let $F$ be a lower AC field, and $F^{+}$be the corresponding superfield. Let us consider the Cauchy problems

$$
\begin{gathered}
\frac{d \boldsymbol{x}}{d t}=F(\boldsymbol{x}), \quad \boldsymbol{x}(0)=u, \\
\frac{d \boldsymbol{x}^{+}}{d t}=F^{+}\left(\boldsymbol{x}^{+}\right), \quad \boldsymbol{x}^{+}(0)=u^{+},
\end{gathered}
$$

such that $u<u^{+}$. Then the corresponding solutions $\boldsymbol{x}, \boldsymbol{x}^{+}$satisfy the estimate

$$
\boldsymbol{x}(t)<\boldsymbol{x}^{+}(t)
$$

for all $t$ such that $\boldsymbol{x}^{+}(t), \boldsymbol{x}(t)$ are defined and lie in $D$.

Similarly, let $F$ be an upper $A C$ field, and $F^{-}$be the corresponding subfield. Let us consider the Cauchy problem

$$
\frac{d \boldsymbol{x}^{-}}{d t}=F^{-}\left(\boldsymbol{x}^{-}\right), \quad \boldsymbol{x}^{-}(0)=u^{-}
$$

such that $u_{-}<u$. Then the corresponding solutions $\boldsymbol{x}, \boldsymbol{x}^{-}$satisfy the estimate

$$
\boldsymbol{x}(t)>\boldsymbol{x}^{-}(t)
$$

for all $t$ such that $\boldsymbol{x}(t), \boldsymbol{x}^{-}(t)$ are defined and lie in $D$.

Proof. We prove the estimate $\boldsymbol{x}(t)<\boldsymbol{x}^{+}(t)$, the second estimate can be derived in a similar way. Suppose that, at a time moment, this inequality is invalid. Then there exist a time moment $t_{0}$ and an index $j$ such that

$$
\begin{gathered}
x_{i}(t) \leq x_{i}^{+}(t) \quad t<t_{0}, \quad i=1, \ldots, n \\
x_{j}\left(t_{0}\right)=x_{j}^{+}\left(t_{0}\right),
\end{gathered}
$$

and

$$
\frac{d x_{j}(t)}{d t} \geq \frac{d x_{j}^{+}(t)}{d t} \quad t=t_{0}
$$

Consider the equations for $j$-th components at $t=t_{0}$. We have

$$
\begin{gathered}
\frac{d x_{j}(t)}{d t}=G_{j}(\boldsymbol{x})-x_{j} q_{j}(\boldsymbol{x}), \\
\frac{d x_{j}^{+}(t)}{d t}=G_{j}^{+}\left(\boldsymbol{x}^{+}\right)-x_{j}^{+} q_{j}^{-}\left(\boldsymbol{x}^{+}\right) .
\end{gathered}
$$

Let us denote $w=x_{j}^{+}-x_{j}$. Then, due to (3.25) and (3.26), one obtains

$$
w\left(t_{0}\right)=0, \quad \frac{d w}{d t}\left(t_{0}\right) \leq 0 .
$$

On the other hand, by (3.27) and (3.28) we have

$$
\frac{d w(t)}{d t}=S_{1}+S_{2}
$$

where

$$
S_{1}=G_{j}^{+}\left(\boldsymbol{x}^{+}\right)-G_{j}(\boldsymbol{x}), \quad S_{2}=x_{j}\left(t_{0}\right)\left(q_{j}\left(\boldsymbol{x}\left(t_{0}\right)\right)-q_{j}^{-}\left(\boldsymbol{x}^{+}\left(t_{0}\right)\right)\right) .
$$

We have $S_{1}\left(t_{0}\right) \geq 0$ by definition of $G_{j}^{+}$and AC fields, see (3.2). Furthermore, by (3.3) $S_{2}>0$. Therefore, we have obtained a contradiction and the assertion is proved. 
Example. Let us consider system (3.6)-(3.13) and let $k_{16}<k_{18}$. Then this system has an absorbing set in the simplex

$$
\mathcal{A}=\left\{\boldsymbol{x}: x_{i} \geq 0, \sum_{j=1}^{8} x_{i}=C\right\},
$$

where $C$ is a constant. This fact allows us to estimate solutions to (3.6)-(3.13) for large $t$ by Prop. 3.3. To obtain an upper estimate, we replace equations (3.10) and (3.11) to

$$
\begin{aligned}
& \frac{d x_{5}}{d t}=k_{4} x_{3}-\left(k_{6}+k_{12}\right) x_{5}+k_{15} x_{7}, \\
& \frac{d x_{6}}{d t}=k_{5} x_{4}-\left(k_{7}+k_{13}\right) x_{6}+k_{16} x_{7},
\end{aligned}
$$

and consider the system of equations (3.6)-(3.9), (3.31), (3.32), (3.12) and (3.13). This modified system is cooperative and thus all local attractors of this system are equilibria $\boldsymbol{X}^{+}$. These equilibria can be found in a simple way since this system contains a single nonlinear term (see the next section). So, for large $t$ solutions of (3.6)-(3.13) satisfy $x_{i}(t)<X_{i}^{+}$.

\section{Systems with sparse quadratic terms}

System (3.6)-(3.13) has a unique nonlinear term. Such systems can be reduced to a single equation with time delay. In some cases this equation can be investigated. Let us consider the following class of vector fields $F_{i}$

$$
F_{i}(\boldsymbol{x})=\sum_{j=1}^{n} M_{i j} x_{j}+a_{i} x_{i_{0}} x_{j_{0}}+k_{i},
$$

where $i_{0}, j_{0} \in\{1, \ldots, n\}$ are some fixed indices, $k_{i}>0, a_{i}$ are some coefficients $M_{i j}>0$ if $j \neq i$.

Let us set $Q(t)=x_{i_{0}}(t) x_{j_{0}}(t), \boldsymbol{a}=\left(a_{1}, \ldots, a_{n}\right)$ and $\boldsymbol{k}=\left(k_{1}, \ldots, k_{n}\right)$. For example, in (3.6)-(3.13) we have $i_{0}=5, j_{0}=6$. Let $M$ be the linear operator defined by matrix $\mathbf{M}$. Let us consider the evolution semigroup $L(t)=\exp (M t)$. Assume that

$$
\|L(t) x\| \leq C \exp (-b t)\|x\|, \quad C, b>0
$$

for all positive $x$ from the positive cone, i.e., such that $x_{i}>0$. Inequality (4.2) holds, for example, if the matrix $M$ has a dominant negative diagonal, i.e.,

$$
\sum_{j=1, \ldots, n, j \neq i} M_{i j}+M_{i i}<0
$$

Then all eigenvalues of $M$ have negative real parts. Moreover, we have the following result.

Lemma 4.1. Assume for (1.1) with $F$ defined by (4.1) there are coefficients $c_{i}>0$ such that $E$ defined by (3.14) satisfies (3.15) with $k_{0}=0$. Then (4.2) holds.

Proof. Relation (3.15) with $k_{0}=0$ implies that $E(t) \leq\|x\| \exp \left(-a_{0} t\right)$. Since $x_{i}>0$, this inequality entails (4.2).

Let us introduce projection operators $P_{i}$ to $i$-th components. Then system (4.1) reduces to the relationship

$$
Q(t)=R_{t}(Q(\cdot))
$$


where a nonlinear operator $R_{t}$ is defined by

$$
R_{t}=\left(\int_{0}^{t} P_{i_{0}} \exp (M(t-\tau))(\boldsymbol{a} Q(\tau)+\boldsymbol{k}) d \tau\right)\left(\int_{0}^{t} P_{j_{0}} \exp (M(t-\tau))(\boldsymbol{a} Q(\tau)+\boldsymbol{k}) d \tau\right) .
$$

This equation, in general, can describe a complicated behaviour (see [12]). A general approach to such equations is to find equilibria and study bifurcations [12]. Assume (4.2) holds. Then equilibria $Q_{*}$ can be found by the following procedure. Given a $Q_{*}$, equations for equilibria give a linear system

$$
\sum_{j=1}^{n} M_{i j} x_{j}=-a_{i} Q_{*}-k_{i},
$$

with respect to $x_{1}, x_{2}, \ldots, x_{n}$. Solving this system, we obtain $x_{i}=\alpha_{i} Q_{*}+\beta_{i}$, where $\alpha_{i}, \beta_{i}$ are some coefficients. Therefore, for $Q_{*}$ we have

$$
Q_{*}=\left(\alpha_{i_{0}} Q_{*}+\beta_{i_{0}}\right)\left(\alpha_{j_{0}} Q_{*}+\beta_{j_{0}}\right) .
$$

Similarly, we can seek equilibria for systems with sparse quadratic terms that leads to a quadratic system of a low dimension.

Let us consider system (3.6)- (3.13) as an example. Here $i_{0}=5$ and $j_{0}=6$. As a result of straightforward computations, we obtain equation (4.5) with

$$
\begin{gathered}
\alpha_{5}=-\left(k_{14}+k_{15} r\right) R_{5}^{-1}, \quad \beta_{5}=R_{5}^{-1} k_{4}\left(k_{2} k_{0}\left(k_{0}+k_{8}\right)^{-1}+k_{4}+k_{10}\right)^{-1} k_{22} k_{8}\left(k_{0}+k_{8}\right)^{-1}, \\
\alpha_{6}=-\left(k_{14}+k_{16} r\right) R_{6}^{-1}, \quad \beta_{6}=R_{6}^{-1} k_{5}\left(k_{3} k_{1}\left(k_{1}+k_{8}\right)^{-1}+k_{3}+k_{11}\right)^{-1} k_{23} k_{1}\left(k_{1}+k_{9}\right)^{-1}, \\
\text { where } r=\left(k_{15}+k_{18}+k_{16} k_{19}\left(k_{17}+k_{19}\right)^{-1}\right)^{-1} \text { and } \\
R_{5}=k_{6}+k_{12}-k_{6} k_{4}\left(k_{2} k_{8}\left(k_{0}+k_{8}\right)^{-1}+k_{4}+k_{10}\right)^{-1}, \\
R_{6}=k_{7}+k_{13}-k_{7} k_{5}\left(k_{3} k_{9}\left(k_{1}+k_{9}\right)^{-1}+k_{3}+k_{11}\right)^{-1} .
\end{gathered}
$$

One can show that in this case equation (4.5) has two positive solutions.

\section{Strategy to find a complex behaviour}

5.1. Realization of vector fields. In this section we state a method of the realization of vector fields (RVF) that allows us to show that the quadratic systems can exhibit complicated large time behaviour. The main idea is to study families of dynamical systems depending on parameters. The complexity of the behaviour of the family can be measured as follows: which vector fields can be obtained by variations of the family parameters. The method RVF has been introduced by P. Poláčik [27, 24, 25] (we change slightly the original version to adapt it for our goals).

Let us consider a family of global semiflows $S_{\mathcal{P}}^{t}$ in a fixed Banach space $B$. We assume that these semiflows depend on a parameter $\mathcal{P} \in B_{1}$, where $B_{1}$ is another Banach space.

Denote by $\mathcal{B}^{m}$ the unit ball $\{q:|q| \leq 1\}$ in $\mathbf{R}^{m}$ centered at 0 , where $q=\left(q_{1}, q_{2}, \ldots, q_{m}\right)$ and $|q|^{2}=q_{1}^{2}+\ldots+q_{m}^{2}$. Let us consider a system of differential equations on the ball $\mathcal{B}^{m}$ :

$$
\frac{d q}{d t}=Q(q)
$$

where

$$
Q \in C^{1}\left(\mathcal{B}^{m}\right), \sup _{q \in \mathcal{B}^{m}}|\nabla Q(q)|<1
$$


Assume the vector field $Q$ is directed strictly inward to the ball $\mathcal{B}^{m}$ at the boundary $\partial \mathcal{B}^{m}=\{q:|q|=1\}:$

$$
Q(q) \cdot q<0, \quad q \in \partial \mathcal{B}^{m} .
$$

Then eq. (5.1) defines a global semiflow on $\mathcal{B}^{m}$. Let $\epsilon$ be a positive number.

Definition 5.1. We say that the family of global semiflows $S_{\mathcal{P}}^{t}$ realizes the vector field $Q$ with accuracy $\epsilon$ (briefly, $\epsilon$-realizes), if there exists a parameter $\mathcal{P}=\mathcal{P}(Q, \epsilon, n) \in B_{1}$ such that

1 semiflow $S_{\mathcal{P}}^{t}$ has a positively invariant manifold $\mathcal{M}_{m}$;

2 this manifold is embedded into $B$ by a sufficiently smooth map

$$
z=Z(q), \quad q \in \mathcal{B}^{m}, \quad z \in B, \quad Z \in C^{1+r}\left(\mathcal{B}^{m}\right),
$$

where $r>0$;

3 the restriction of the semiflow $S_{\mathcal{P}}^{t}$ to $\mathcal{M}_{m}$ is defined by the system of differential equations

$$
\frac{d q}{d t}=Q(q)+\tilde{Q}(q, \mathcal{P}), \quad Q \in C^{1}\left(\mathcal{B}^{m}\right)
$$

where

$$
|\tilde{Q}(\cdot, \mathcal{P})|_{C^{1}\left(\mathcal{B}^{m}\right)}<\epsilon .
$$

This means that the dynamics on the invariant manifold is defined by the variables $q_{1}, q_{2}, \ldots, q_{m}$ and approximates prescribed dynamics (5.1) with accuracy $\epsilon$.

Using these ideas let us describe a realization vector field approach for systems (1.1), where the field $\mathbf{F}$ is defined by (1.2). This approach works under some assumptions, which mean that the system involves slow and fast variables. To avoid too cumbersome relations, we assume $K_{i j k}=0$ for all $i, j, k$.

Let us choose a subset $K_{m}=\left\{i_{1}, \ldots, i_{m}\right\}$ of $I_{n}=\{1,2, \ldots, n\}$, where $1 \leq m<n$. Let $\bar{K}_{m}=I_{n}-K_{m}$ be the complement of $K_{m}$. We break all variables $x_{i}$ into two sets, the slow ones $q_{j}=x_{i_{j}}$ and the fast variables, which are all the rest $x_{k}$ with $k \in \bar{K}_{m}$.

In the new variables system (1.1) takes the form

$$
\frac{d q_{l}}{d t}=b_{i_{l}}+\sum_{j \in \bar{K}_{m}} M_{i_{l} j} x_{j}+\sum_{s=1}^{m} M_{i_{l} i_{s}} q_{s}-\left(\tilde{b}_{i_{l}}+\sum_{k \in \bar{K}_{m}} b_{i_{l} k} x_{k}+\sum_{s=1}^{m} b_{i_{l} i_{s}} q_{s}\right) q_{l}
$$

where $l=1, \ldots, m$

$$
\frac{d x_{i}}{d t}=b_{i}+\sum_{j \in \bar{K}_{m}} M_{i j} x_{j}+\sum_{s=1}^{m} M_{i i_{s}} q_{s}-\left(\tilde{b}_{i}+\sum_{k \in \bar{K}_{m}} b_{i k} x_{k}+\sum_{s=1}^{m} b_{i i_{s}} q_{s}\right) x_{i}
$$

where $i \in \bar{K}_{m}$.

Furthermore, let $\lambda>0$ be a large parameter. Assume that

$$
b_{i_{l}}=\lambda^{-1} a_{l}, \quad M_{i_{l} i_{s}}=\lambda^{-1} \mu_{l s}, \quad \tilde{b}_{i_{l}}=\lambda^{-1} \tilde{a}_{l}, \quad b_{i_{l} i_{s}}=\lambda^{-1} \beta_{l s},
$$

for all $l, s=1, \ldots, m$ and

$$
\tilde{b}_{i}=\lambda \bar{b}_{i}, \quad b_{i i_{s}}=\lambda \bar{b}_{i s}, \quad i=1, \ldots, n, s=1, \ldots, m .
$$

We suppose that

$$
\left|a_{l}\right|,\left|\tilde{a}_{l}\right|,\left|\mu_{l s}\right|\left|\beta_{l s}\right|,\left|M_{i_{l} j}\right|,\left|b_{i_{l} k}\right|<C_{1},
$$


where $i, s=1, \ldots, m$ and $j, k \in \bar{K}_{m}$,

$$
\left|\bar{b}_{i}\right|,\left|M_{i j}\right|,\left|M_{i i_{s}}\right|,\left|\bar{b}_{i}\right|,\left|b_{i k}\right|\left|\bar{b}_{i s}\right|<C_{2},
$$

where $i, k \in \bar{K}_{m}, s=1, \ldots, m$ and the constants $C_{i}$ are independent of $\lambda$. Let $\mathcal{B}_{R}^{m}=\{q$ : $|q|<R \mid\}$ be a ball in $\mathbb{R}^{m}$ of the radius $R$ centered at 0 .

Then, by classical theorems of the invariant manifolds $[\mathbf{1 4}, \mathbf{1 1}, \mathbf{4 0}, \mathbf{4 3}]$ we obtain the following assertion.

Lemma 5.2. Assume that conditions (5.11) and (5.12) hold. Then for sufficiently large $\lambda>\lambda_{0}\left(C_{1}, C_{2}, R\right)$ there exists a locally invariant and locally attracting $m$-dimensional manifold $\mathcal{M}_{m}$ defined by

$$
x_{i}=\lambda^{-1} X_{i}(q)+\lambda^{-2} \phi_{i}(q, \lambda), \quad i \in \bar{K}_{m}, \quad q \in \mathcal{B}_{R}^{m}
$$

where

$$
X_{i}(q)=\left(b_{i}+\sum_{s=1}^{m} M_{i i_{s}} q_{s}\right)\left(\bar{b}_{i}+\sum_{s=1}^{m} \bar{b}_{i i_{s}} q_{s}\right)^{-1}, \quad i \in \bar{K}_{m}
$$

and where functions $\phi_{i}$ satisfy estimates

$$
\left|\phi_{i}\right|_{C^{1}\left(\mathcal{B}_{R}^{m}\right)}<C_{0}, \quad i=1, \ldots, n .
$$

Dynamics (1.1) restricted to $\mathcal{M}_{m}$ is defined by the system

$$
\frac{d q_{l}}{d t}=\lambda^{-1} Q_{l}(q)+\lambda^{-2} \tilde{Q}_{i}(q, \lambda)
$$

where

$$
Q_{l}(q)=a_{l}+\sum_{j \in \bar{K}_{m}} M_{i_{l} j} X_{j}(q)+\sum_{s=1}^{m} \mu_{l s} q_{s}-\left(\tilde{a}_{l}+\sum_{j \in \bar{K}_{m}} b_{i_{l} j} X_{j}(q)+\sum_{s=1}^{m} \beta_{l s} q_{s}\right) q_{l}
$$

and

$$
\mid \tilde{Q}_{i}\left(\cdot,\left.\lambda\right|_{C^{1}\left(\mathcal{B}_{R}^{m}\right)}<C_{14}, \quad i=1, \ldots, n .\right.
$$

This lemma follows, for example, from results [14], Theorems 6.1 and 6.9.

Let us investigate sensitivity of the inertial form $Q(q)$ on parameters. Consider the term $G_{i}(q)=\sum_{j \in \bar{K}_{m}} M_{i_{l} j} X_{j}(q)$ involved in expression (5.17) for $Q_{i}$. We have

$$
G_{i}(q)=\sum_{j \in \bar{K}_{m}} M_{i_{l} j}\left(b_{j}+\sum_{s=1}^{m} M_{j i_{s}} q_{s}\right)\left(\bar{b}_{j}+\sum_{s=1}^{m} \bar{b}_{j i_{s}} q_{s}\right)^{-1} .
$$

For large $n$ this term has a property of stability with respect to random variations of coefficients $M_{i_{l} j}, M_{j i_{s}}, \bar{b}_{j}$ and $\bar{b}_{j i_{s}}$.

Assume that these coefficients are random variables subject to independent distributions and such that their expectations and variances fulfill estimates

$$
\left|E M_{i_{l} j}\right|,\left|E M_{j i_{s}}\right|,\left|E \bar{b}_{j}\right|,\left|E b_{j i_{s}}\right|<C_{E},
$$

and

$$
\left|\operatorname{Var}\left(M_{i_{l}}\right)\right|,\left|\operatorname{Var}\left(M_{j i_{s}}\right)\right|,\left|\operatorname{Var}\left(\bar{b}_{j}\right)\right|,\left|\operatorname{Var}\left(b_{j i_{s}}\right)\right|<C_{v},
$$

where $C_{E}, C_{v}$ are positive constants independent on $\lambda$ and $n$. 
Then for $q \in B_{R}$ we have the following relations

$$
E G_{i}(q)=\sum_{j \in \bar{K}_{m}} E M_{i_{l} j}\left(E b_{j}+\sum_{s=1}^{m} E M_{j i_{s}} q_{s}\right) E\left(\bar{b}_{j}+\sum_{s=1}^{m} \bar{b}_{j i_{s}} q_{s}\right)^{-1}
$$

and

$$
\left|\operatorname{Var}\left(G_{i}(q)\right)\right|<C n,
$$

where a positive constant $C$ is independent of $n$.

These relations demonstrate robustness of the terms $G_{i}(q)$ with respect to random variations of the coefficients $M_{i_{l} j}, M_{j i_{s}}, \bar{b}_{j}$ and $\bar{b}_{j i_{s}}$. In fact, generally the expectations $E G_{i}(q)$ have order $O(n)$ whereas the corresponding deviations $\operatorname{Var}\left(G_{i}(q)\right)^{1 / 2}$ are $O\left(n^{1 / 2}\right)$.

Finally we conclude that reduced few dimensional dynamics is robust with respect to variations of the coefficients $M_{i_{l} j}, M_{j i_{s}}, \bar{b}_{j}$ and $\bar{b}_{j i_{s}}$. However, this dynamics may be sensitive with respect to variations of the coefficients $a_{l}, \tilde{a}_{l}$ and $\beta_{l s}$.

5.2. Complicated behaviour for large times. Let us consider systems (1.1) with $F_{i}$ defined by (1.2). For the case when the coefficients $b_{i k}<0$, these systems generate cooperative dynamics. Then the large time behaviour is relatively simple. We show that in the case $b_{i k}>0$ and large $n$ we can obtain a complicated dynamics, in particular, all kinds of hyperbolic dynamics. It is well known that such dynamics may be periodic or even chaotic $[\mathbf{2}, \mathbf{2 9}, \mathbf{1 7}, \mathbf{2 8}]$. We assume that the conditions $b_{i k}>0, M_{i j}>0$ and $K_{i j k}=0$ hold for all $i, j, k$ therefore the corresponding system (1.1) may be almost cooperative.

TheOREm 5.3. Consider system (5.1) defined on the unit ball $\mathcal{B}^{m}$ satisfying condition (5.2). Assume that the global semiflow $S_{Q}^{t}$ defined by this system has a hyperbolic set $H_{Q}$. Let $\epsilon$ be a small positive number. Then there exist a number $n$ and coefficients $b_{i}, M_{i j}, \tilde{b}_{i}, b_{i k}$ and $K_{i j k}$ such that the following property holds.

Consider the field $F$ defined by these coefficients by (1.2). Consider the semiflow $S_{F}^{t}$ generated by the corresponding system (1.1). Then this semiflow also has a hyperbolic set $H_{F}$ homeomorphic to $H_{Q}$. The corresponding restricted dynamics $\left.S_{Q}^{t}\right|_{H_{Q}}$ and $\left.S_{F}^{t}\right|_{H_{F}}$ are topologically orbitally equivalent.

Proof. We set $K_{i j k}=0, b_{i}=0$ and $M_{i j}=0$ for all $i, j, k$. Then system (1.1) becomes a generalized Lotka-Volterra one. We consider the number $n$ and the coefficients $\tilde{b}_{i}$ and $b_{i k}$ as a parameter $\mathbf{P}$. Now this theorem follows from results [38].

Such proving method has, however, a drawback. The hyperbolic sets that can be constructed by this reduction to the generalized Lotka-Volterra systems lie on unstable invariant manifolds $\mathcal{M}_{m}$. Therefore, the corresponding hyperbolic dynamics is unstable with respect to variations of initial data (when these data leave the invariant manifold).

We can overcome this difficulty using the results of the previous section and the following approximation lemma. We set $K_{i j k}=0$ for all $i, j, k$ and extend the set of parameters including in $\mathbf{P}$ the number $n$ and all coefficients $\left.b_{i}, M_{i j}, \tilde{b}_{i}, b_{i k}\right\}$. Let $K_{m}=\{1, \ldots, m\}$.

Lemma 5.4. Consider the family of vector fields on the unit ball $\mathcal{B}^{m}$ depending on parameter $\mathbf{P}$ :

$$
V_{i}(q, \mathbf{P})=\sum_{j \in \bar{K}_{m}} M_{i_{l} j}\left(b_{j}+\sum_{s=1}^{m} M_{j i_{s}} q_{s}\right)\left(\bar{b}_{j}+\sum_{s=1}^{m} \bar{b}_{j i_{s}} q_{s}\right)^{-1}
$$


For any $C^{1}$-smooth field $Q(q)$ on $\mathcal{B}^{m}$ and any $\epsilon>0$ there exists a $\mathbf{P}$ such that

$$
|V(\cdot, \mathbf{P})-F(\cdot)|_{C^{1}\left(\mathcal{B}^{m}\right)}<\epsilon
$$

Moreover, conditions (1.3) and $b_{i k}>0 \forall i, k$ hold.

We prove this lemma for $m=1$. The general case of arbitrary $m$ can be obtained from the one dimensional case by the standard methods, see [3]. For $m=1$ we set $q_{1}=z$, $M_{i_{1} j}=M_{j}$ and $\bar{b}_{j i_{s}}=M_{j i_{s}}=a_{0}$. Let $a_{0}>0$ be fixed, the number $n$ and the positive coefficients $M_{j}, \bar{b}_{j}$ and $b_{j}$ will be considered as parameters. Relation (5.24) takes the form

$$
V(z, \boldsymbol{h}, c)=c+\sum_{j=2}^{n} \rho_{j}\left(h_{j}+z\right)^{-1} .
$$

where $\rho_{j}=a_{0}^{-1} M_{j}\left(b_{j}-\bar{b}_{j}\right), c=\sum_{j=2}^{n} M_{j}$ and $h_{j}=b_{j} / a_{0}$. Note that the coefficients $\rho_{j}$ can have different signs even under conditions $M_{j}>0, b_{j}>0, \bar{b}_{j}>0$ and $a_{0}>0$.

Let us show that the set $W$ of the functions $V$ defined by relation (5.26), where $\left|h_{j}\right| \leq 1$, is dense in the space of continuously differentiable functions on $I=[0,1]$, i.e., $C \operatorname{Clos}(W)=C^{1}(I)$. Notice that, due to the Weierstrass theorem, for each $\epsilon_{1}>0$, each function $g(z)$ from $C^{1}(I)$ can be approximated by a sum $P(z)=\sum_{k=1}^{n} p_{k}(1+z)^{-k}$ with accuracy $\epsilon_{1}$ :

$$
|g-P|_{C^{1}(I)}<\epsilon_{1}
$$

Now, by an inductive procedure, we show that each function $p_{k}(z)=(1+z)^{-k}$ can be approximated in $C^{1}$ - norm by a sum (5.26). In fact, let

$$
S(z, \delta)=\delta^{-1}\left((1+z)^{-1}-(1+(z-\delta))^{-1}\right) .
$$

Then

$$
\sup _{z \in I}\left|(1+z)^{-2}-S(z, \delta)\right|<c_{1} \delta, \quad \sup _{z \in I}\left|\frac{d}{d z}\left((1+z)^{-2}-S(z, \delta)\right)\right|<c_{2} \delta .
$$

In a similar way, we can approximate $(1+z)^{-3},(1+z)^{-4}$ etc.

To prove the lemma for $m>1$, one use a standard trick, namely, the Radon transformation, see $[\mathbf{3}]$. Indeed, any functions $F(\boldsymbol{q})$ can be approximated by a linear combination of the functions $F_{\boldsymbol{c}}(\boldsymbol{q})=f\left(z_{\boldsymbol{c}}\right)$ of the scalar arguments $z_{\boldsymbol{c}}$, where $z_{\boldsymbol{c}}=\boldsymbol{c} \cdot \boldsymbol{q}=$ $c_{1} q_{1}+c_{2} q_{2}+\ldots+c_{m} q_{m}$.

This lemma proves the theorem as follows. The lemma shows that the semiflow $S_{F}^{t}$ $\epsilon$-realizes any $m$-dimensional $C^{1}$ - smooth vector field, in particular, the field $Q$. If we choose $\epsilon$ small enough, then, using the definition of structural stability and the theorem on structural stability of hyperbolic sets (see $[\mathbf{1 7}, \mathbf{2 8}, \mathbf{2 9}]$ and subsections 1.2 and 2.3), we obtain our assertion.

\section{Conclusion}

We have considered a class of systems of differential equations with quadratic nonlinearities. This class have important applications for biochemistry and population dynamics, in particular, generalized Lotka-Volterra systems belong to this class.

We have also introduced a general class of systems of differential equations, the almost cooperative systems (AC systems). Under some natural conditions, quadratic systems are almost cooperative. We have obtained estimates of solutions for AC systems. By purely 
algebraic methods, that can be realized algorithmically by an elementary linear algebra, we have studied the following problems :

(i) Finding invariant boxes in the phase space;

(ii) finding equilibria;

(iii) how to construct reductions of complicated high dimensional systems to low dimensional ones.

These reductions allow us to find periodical and chaotic regimes for almost cooperative quadratic systems. Due to a transparent structure of the reduction algorithm, we can investigate sensitivity of reduced low dynamical systems with respect to system parameter variations. We show that the random variations of many parameters do not affect reduced dynamics.

\section{Acknowledgements}

The first and third authors have made this work in the frames of a DFG grant. The second author is grateful to the Max-Planck Institut für Mathematik, Bonn for its hospitality. Also the first author was financially supported by Government of the Russian Federation, Grant 074-U01. We are grateful to Referees for interesting comments, which significantly improved the article.

\section{References}

[1] A. A. Andronov and L. S. Pontryagin. Structurally stable systems. Dokl. Akad. Nauk SSSR, 14(5):247250, 1937.

[2] V. I. Arnol'd, Geometric methods in Theory of Ordinary Differential Equations, 2nd ed. Springer, New York, 1988.

[3] A. Barron, (1993) Universal Approximation Bounds for superpositions of a sigmoidal functions, IEEE Trans. on Inf. Theory, 39, 930-945.

[4] Dynamical Systems with Hyperbolic Behaviour, D. V. Anosov (ed). (Dynamical Systems 9). Encyclopedia of Mathematical Sciences Vol. 66. Translated from Russian., Springer V., Berlin, Heidelberg, New-York. 1995.

[5] B. Banhelyi, T. Csendes, B. M. Garay, and L. Hatvani. A computer-assisted proof of 3-chaos in the forced damped pendulum equation. SIAM Journal on Applied Dynamical Systems, 7(3):843867, 2008.

[6] R. Csikja and J. Toth. Blow up in polynomial differential equations. En- formatika. International Journal of Applied Mathematics and Computer Sciences, 4(2):728733, 2007.

[7] Fenichel N. Persistence and smoothness of invariant manifolds for flows, Indiana Univ. Math. 1971, Vol 21., pp. 193-225.

[8] B.M. Garay, Discretization and Morse Smale dynamical systems on planar discs, Acta Math. Univ. Comenianieae, Vol. LXIII, pp. 25-38, (1994).

[9] L. Grune (2002) Asymptotic behaviour of dynamical and control systems under perturbation and discretization, Lecture Notes in Mathematics, Springer.

[10] J. Hofbauer and K. Sugmund, Evolutionary games and population dynamics, 1988. Cambridge University Press.

[11] J. Guckenheimer and P.Holmes, Nonlinear Osscillations, Dynamical Systems, and Bifurcations of Vector Fields, Springer, New York (1981).

[12] J. K. Hale, "Asymptotic behavior of dissipative systems", American Mathematical Society, Providence, 1988.

[13] A. Halmschlager, L. Szenthe, and J. Tóth, Invariants of kinetic differential equations, Electronic Jounral of Qualitative Theory of Differential equations, Proc. 7th Colloc. QTDE, 2004, No. 14, 1-14.

[14] D. Henry, Geometric theory of semilinear parabolic equations, Lecture Notes in Mathematics, Vol. 840, Springer, Berlin. 1981.

[15] M. W. Hirsch, Stability and convergence in strongly monotone dynamical systems, J. Reine. Angew. Math., 1988, 383 pp. $1-58$

[16] Ilyashenko Yu, Weigu Li, Nonlocal bifurcations, Amer. Math. Society, 1999.

[17] A. B. Katok and B. Hasselblatt, Introduction to the Modern Theory of Dynamical Systems, Cambridge University Press, Encyclopedia of Mathematics and Its Applications, Vol.54, 1995.

[18] M. Kinyon and A. A. Sagle, Quadratic dynamical systems and algebras, J. Diff. Eq. 117(1) 1995, no. 1, $67-126$.

[19] P. De Leenheer, D. Angeli, and E. D. Sontag. Monotone chemical reaction networks. J. Math. Chem., 41(3):295314, 2006. 
[20] C. Li, M. Donizelli, N. Rodriguez, N., H. Dharuri, L. Endler, V. Chelliah, L. Li, E. He, A. Henry, M. I. Stefan, J. L. Snoep, M. Hucka, N. Le Novère, C. Laibe: BioModels database: An enhanced, curated and annotated resource for published quantitative kinetic models. BMC Systems Biology 4 (January 2010 ) 92

[21] E. N. Lorenz, Deterministic nonperiodic flow, J. Atmos. Sci, 1963, V20, p.130 -141.

[22] R. Newhouse, D. Ruelle and F. Takens, Occurence of strange axiom A attractors from quasi periodic flows, Comm.Math. Phys., 1971, 64 pp. 35-40.

[23] M. Peixoto, Structural stability on two dimensional manifolds, Topology, 1962, Vol. 1, 101-120.

[24] P. Poláčik, Realization of any finite jet in a scalar semilinear parabolic equation on the ball in $R^{2}$, Annali Scuola Norm Pisa 1991, 17, 83-102.

[25] P. Poláčik, Complicated dynamics in Scalar Semilinear Parabolic Equations, In Higher Space Dimensions J. of Diff. Eq. 1991, 89 pp. 244 - 271.

[26] P. Poláčik, High dimensional $\omega$-limit sets and chaos in scalar parabolic equations, J. Diff. Equations, 1995, 119 pp. 24-53.

[27] P. Poláčik, Parabolic equations: Asymptotic behaviour and Dynamics on Invariant Manifolds, Ch.16, pp. 835-883, in: HANDBOOK OF DYNAMICAL SYSTEMS, VOL 2., Edited by B. Fiedler, 2002.

[28] C. Robinson, Dynamical Systems: Stability, Symbolic Dynamics, and Chaos, CRC Press, 1999.

[29] D. Ruelle, Elements of differentiable dynamics and bifurcation theory, Acad. Press, Boston etc. 1989

[30] D. Ruelle and F. Takens, On the nature of turbulence, Comm. Math. Phys, 1971, 20 pp. 167 -192.

[31] K. P. Rybakowski, Realization of arbitrary vector fields on center manifolds of parabolic Dirichlet BVP's, J. Differential Equations 1994, 114 pp. 199-221.

[32] V. V. Sharko, $L^{2}$ invariants and Morse-Smale flows on manifolds, Ukrain Math. Journal, (2007), Vol. 59, N4, pp. $522-533$.

[33] H. L. Smith and H. R. Thieme, Convergence for strongly order preserving semiflows, SIAM J. Math. Anal., 1991,22 $1081-1101$.

[34] Smoller, J., Shock Waves and Reaction -Diffusion Equations, Springer V., New- York, 1983.

[35] R. Temam, Infinite dimensional dynamical systems in mechanics and physics, Springer, New York 1988.

[36] Tucker, W. (2002). "A Rigorous ODE Solver and Smale's 14th Problem". Found. Comp. Math. 2: 53117. http://www.math.uu.se/ warwick/main/rodes.html.

[37] T. Turanyi. Sensitivity analysis of complex kinetic systems. Tools and applications. J. Math. Chem., $5(3): 203248,1990$.

[38] V. Kozlov, S. Vakulenko, "On chaos in Lotka-Volterra systems: an analytical approach", Nonlinearity, 26(8): 2299-2314, 2013

[39] S. A. Vakulenko, Dissipative systems generating any structurally stable chaos Advances in Differential Equations, 2000, 5, no. 7-9, pp. 1139-1178.

[40] A. Vanderbauwhede and G. Ioss, Center manifold theory in infinite dimensions, Dynamics Reported: Expositions in Dynamical systems, Springer, Berlin (1992), 125-163.

[41] M. Viana, Multidimensional nonhyperbolic attractors, Publications IHES, 1997, 85, 63-96.

[42] A. I. Volpert. Differential equations on graphs. Mat. Sbornik., 88(130):578588, 1972.

[43] S. Wiggins, Normally Hyperbolic Invariant Manifolds in Dynamical Systems, Spinger, New York 1994.

Institute for Mech. Engineering Problems Saint Petersburg, Russia and Saint Petersburg National Research University of Information Technologies, Mechanics and Optics, Saint Petersburg, Russia

CNRS, Mathématiques, Université de Lille, 59655, Villeneuve D’Ascq, France

Bonn University , Germany 